\title{
Molecular Beam Epitaxy Growth for Quantum Cascade Lasers
}

\author{
K. Kosiel*, A. Szerling, J. Kubacka-Traczyk, P. Karbownik, \\ E. Pruszyńska-Karbownik And M. Bugajski \\ Institute of Electron Technology, al. Lotników 32/46, 02-668 Warsaw, Poland
}

\begin{abstract}
The fabrication of quantum cascade lasers emitting at $9 \mu \mathrm{m}$ is reported. The devices operated in pulsed mode at up to $260 \mathrm{~K}$. The peak powers recorded at $77 \mathrm{~K}$ were over $1 \mathrm{~W}$ and the slope efficiency $\eta \approx 0.5-0.6 \mathrm{~W} / \mathrm{A}$ per uncoated facet. This has been achieved by the use of $\mathrm{GaAs} / \mathrm{Al}_{0.45} \mathrm{Ga}_{0.55} \mathrm{As}$ heterostructure, with the "anticrossed-diagonal" design. Double plasmon planar confinement with Al-free waveguide has been used to minimize absorption losses. The double trench lasers were fabricated using standard processing technology, i.e., wet etching and $\mathrm{Si}_{3} \mathrm{~N}_{4}$ for electrical insulation. The quantum cascade laser structures have been grown by molecular beam epitaxy, with Riber Compact $21 \mathrm{~T}$ reactor. The stringent requirements — placed particularly on the epitaxial technology — and the influence of technological conditions on the device structure properties were presented and discussed in depth.
\end{abstract}

PACS numbers: 42.55.Px, 81.15.Hi, 85.60.-q, 85.35.Be, 73.63.-b, 63.22.-m, 72.80.Ey, 73.61.Ey, 78.66.Fd

\section{Introduction}

The quantum cascade lasers (QCLs) are unipolar devices based on tunneling and intersubband transitions, in which the electronic states, wave functions and lifetimes of relevant states are engineered through the quantum mechanical confinement imposed by a complex multilayer structure $[1,2]$. The second main feature of this type of lasers is the cascading scheme of carriers route through the laser active region (AR). That means the single carrier is used more than one time for generating photon at best the number of generated photons equals to the number of QCL modules, through which the carrier is cascaded. For QCLs to work, the extremely precise tailoring of energy levels of quantum states, optical dipole matrix elements, tunneling times and scattering rates of carriers is required. The next crucial issue for laser operation is the concentration of carriers in the structure.

Thus the principle of operation of QCL structures places stringent requirements on the individual layer thickness and composition as well as the overall periodicity of the whole structure. The laser operation is possible only when the designed structure is strictly realized, with the extreme technological precision concerning geometrical and doping features.

The extremely low tolerance of the QCLs for the deviation from the proper geometry does not meet its analogue in the field of technology of semiconductor devices. For the mid-infrared (MIR) emitters the overall thickness tol-

\footnotetext{
* corresponding author; e-mail: kosiel@ite.waw.pl
}

erance seems to be only a few percent. On the basis of our experimental statistics we believe that the thickness tolerance limit is $\approx 3 \%$. For the deviation from original design above this value the devices will not lase. The similar accuracy is required for the composition of layers. For emitters working at longer wavelengths (e.g., in the far-infrared, FIR region) the thickness tolerance is probably even sharper. That is why the technology of epitaxy used for QCL heterostructures must be characterized by perfect precision, long time stability of growth parameters as well as run-to-run reproducibility.

There are some fundamental difficulties concerning QCL structure operation, and most of them are bound to the pumping of the upper laser level in the emitting region, and obtaining the inversion of states. They may result for example from scattering of carriers from the injector ground state directly to the lower laser level, the escape of carriers from upper laser level to the continuum states, from too long lifetime on the lower laser level (poor depopulation of this state) or excessive absorption of radiation by free carriers. In order to minimize these effects several solutions were proposed with respect to $\mathrm{AR} /$ module construction. The main designs are: 3QW (three-level), 4QW (four-level), superlattice and bound-to-continuum constructions. They all, however, are multilayer and composed of ultrathin layers, as well. Again, the necessity of superb epitaxial technology has to be emphasized.

One of the crucial problems of QCLs' operation concerns the heating effect, which is distinctly larger than in the state of the art bipolar lasers. It is connected with the "natural" processes of depopulation of the lower laser 
level and carrier thermalization in the injector, by scattering with the optical or acoustic phonons. The heating results in the higher threshold and operation currents of the lasers, and all this in turn results in the necessity of the effective heat extraction. The problem of heating is intensified in QCLs because of the nature of their active regions containing many interfaces, and layers with thicknesses similar to the mean free path of phonons, which causes that the heat dissipation is strongly hampered.

Naturally the solution of the complex problems of QCLs construction and technology has to be found in all constituent fields, i.e., also in the case of appropriate waveguides and resonators. The problem of exact realization of QCL active region remains however the matter of the most stringent tolerance limits of this device, and hence the biggest challenge for its technology at all.

The physical basis of QCLs operation principle is fundamentally different from that of the "classical" bipolar semiconductor lasers, in which the emission is due to the interband radiative recombination of pairs of carriers instead of intersubband transitions which lead to lasing in QCLs. This fact influences the shape of gain as a function of quantum energy. Hence, the quantum cascade lasers with their narrow spectral lines, resemble the emitters which are based on electron transitions between quantum energy levels in individual atoms, i.e., the gas lasers or solid-state lasers.

The available QCL emitter wavelength range is from $3.5 \mu \mathrm{m}$ up to $250 \mu \mathrm{m}$, so it generally covers the very wide IR spectrum, from MIR up to FIR. In comparison with the performance of bipolar lasers, this is about a two orders of magnitude increase of the wavelength range available for semiconductor lasers, towards the longer wavelengths, with the exclusion of the so-called Reststrahlen region of radiation (i.e., $30-60 \mu \mathrm{m}$ ), where the photon energies match the optical phonon energies - and hence the radiation is absorbed by the semiconductor material. The huge spectral flexibility of the emission is a result of the used intraband generation mechanism.

QCLs may be used in free-space communication systems, molecular spectroscopy and imaging, for science, biomedicine, military purposes and public security. Their wavelength range covers the two optical windows (3-5 $\mu \mathrm{m}, 8-14 \mu \mathrm{m})$. On the other hand, there is a variety of wavelengths absorbed by gases (e.g. by $\mathrm{NO}, \mathrm{CH}_{4}, \mathrm{CO}_{2}$, $\mathrm{CO}$ ), in the range of $3.5-24 \mu \mathrm{m}$. The very wide spectral range as well as the possible wavelength control (obtained in distributed feedback lasers, DFBs) and wavelength tunability (for example by temperature or supply current changes) of QCLs are their great advantage.

The fast development in the field of QCLs is clearly observable, as for example the first continuous wave (CW) (operating at cryogenic temperatures) as well as the first room-temperature lasers have been demonstrated just a year after constructing the first QCL. Again, roughly one-year periods separate the events of obtaining the first DFB-QCL laser, the first superlattice-QCL (SL-QCL), the first trace gas sensing made with the use of DFB-
-QCL, the first mode-locked QCL, and the successful increase of the lasing wavelength beyond $20 \mu \mathrm{m}$.

In this paper we analyse in detail the MBE growth technology applied for preparation of $\mathrm{Al}_{0.45} \mathrm{Ga}_{0.55} \mathrm{As} / \mathrm{GaAs}$ QCL heterostructures. We present the development of this technology up to the final result, and discuss the influence of technological conditions on the lasers properties.

\section{MBE technology for the $\mathrm{Al}_{0.45} \mathrm{Ga}_{0.55} \mathrm{As} / \mathrm{GaAs}$ QCL heterostructures}

\subsection{Construction of the heterostructure}

The AR of the laser structure studied in this paper (Fig. 1) was the 36 period sequence of modules made of $\mathrm{Al}_{0.45} \mathrm{Ga}_{0.55} \mathrm{As} / \mathrm{GaAs}$ coupled quantum wells. The application of relatively high $\mathrm{Al}$ contents in AlGaAs layers, and hence the significant height of barriers improves the thermal performance of the devices [3], in comparison with the earlier AlGaAs/GaAs QCL construction [4]. Every module was composed of quasi-superlattice injector and emitting region based upon the three-quantum level design (3QW). The electronic band structure of QCL has been calculated by solving Schrödinger equation with position dependent effective mass by finite difference method (FDM) [5] — see Fig. 2. The essential features of the considered design are diagonal anti-crossed transitions [4] from state E3 to E2 and depopulation of the laser ground state $\mathrm{E} 2$ by resonant optical phonon $\left(\mathrm{LO}^{\Gamma}\right)$ emission and tunneling into the injector. The calculated lifetime of the excited state and dipole matrix element are $\tau_{3}=1.4 \mathrm{ps}$, and $z_{32}=1.71 \mathrm{~nm}$, respectively. The ground state $\mathrm{E} 2$ is depopulated in the time $\tau_{2-1} \approx 0.3$ ps [3]. These calculations were done at $48 \mathrm{kV} / \mathrm{cm}$, close to estimated laser threshold.

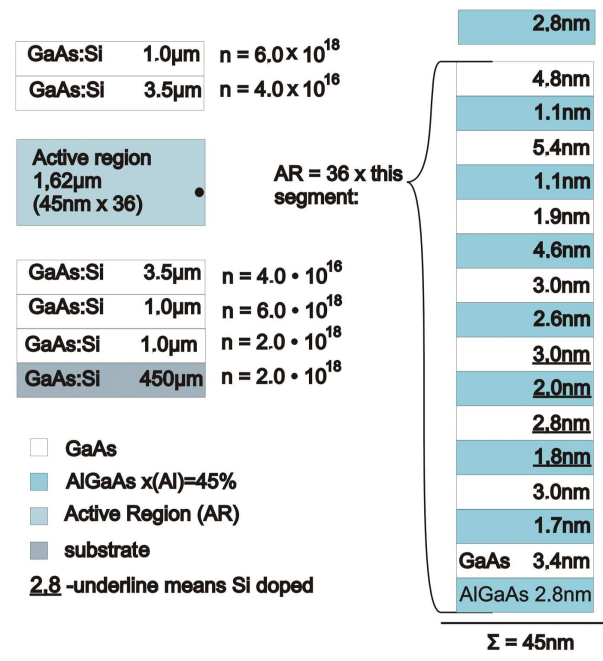

Fig. 1. Layer sequence in the $\mathrm{Al}_{0.45} \mathrm{Ga}_{0.55} \mathrm{As} / \mathrm{GaAs}$ laser structure.

The injector region, apart of its function connected with the relaxation and injection of electrons, plays also 


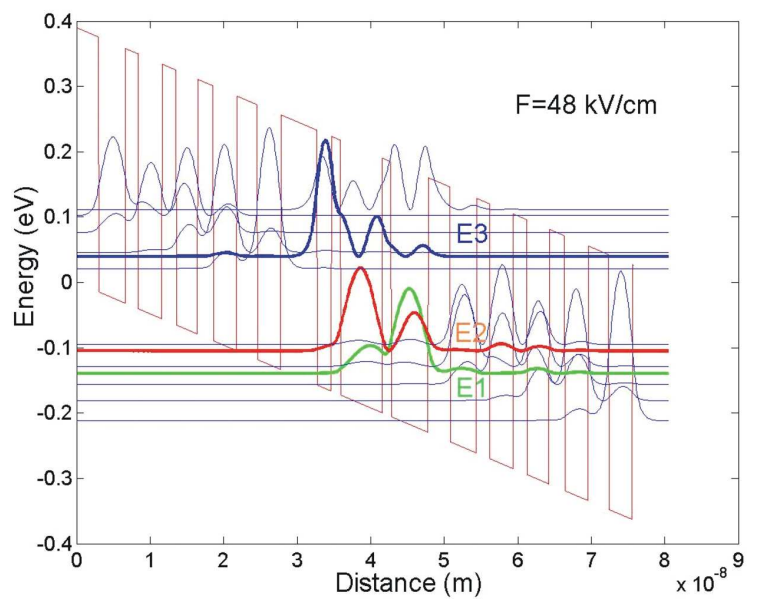

Fig. 2. Conduction band profile and moduli squared wave functions in injector/active/injector segment of the laser under the applied field of $48 \mathrm{kV} / \mathrm{cm}$. The wave functions have been shifted to the energy positions of the respective levels. The E3, E2 and E1 refer to the upper, lower and ground state of lasing transitions. The thin blue lines are the injector lower miniband states. The lowest energy state in the injector couples directly to the upper laser level E3. The topmost state is the $\Gamma$ continuum state. It is located $\approx 80 \mathrm{meV}$ above the upper laser level. The calculated energy differences $E_{32}=147.1 \mathrm{meV}(\lambda=8.4 \mu \mathrm{m})$ and $E_{21}=38.5 \mathrm{meV}$.

the role of a Bragg reflector. This effect is connected with the presence of a "minigap" formed in the injector multi-quantum-well (MQW) structure, which prevents electrons from escaping into the continuum. Only two central, barrier-QW pairs inside each injector have been doped. The tested range of the injector doping was $0.4 \times 10^{12}-2.2 \times 10^{12} \mathrm{~cm}^{-2}$ per period.

The AR was built in the double-plasmon Al-free waveguide region, for planar optical confinement. The waveguide was made of the GaAs, with an appropriate profile of $n$-type doping. Such a waveguide is much less lossy than the one made of the AlGaAs/GaAs system, and based on the refractive index contrast of the involved materials. The main disadvantage of the AlGaAs/GaAs results from the relatively low value of this contrast in the IR wavelength region of interest. The high radiation losses experienced in $\mathrm{AlGaAs} / \mathrm{GaAs}$ waveguide are the result of radiation leakage beyond the AlGaAs cladding region, and from the free-carrier absorption, as the cladding has to be doped. On the contrary, though the Al-free plasmon waveguide is enclosed by the highly doped $\left(6 \times 10^{18} \mathrm{~cm}^{-3}\right)$ GaAs layers, it is, when designed properly, a low-loss one, as it offers very efficient confinement of the IR radiation. The doping level of the GaAs cladding layer was matched to obtain the maximum $n$-type carrier concentration, without any autocompensation effect, which may appear after the critical Si dopant concentration is exceeded.

\subsection{Technological background, modelling and characterization environment}

The laser structures were grown by solid source MBE technology in Riber Compact $21 \mathrm{~T}$ machine. The molecular beams of the group III elements $(6.5 \mathrm{~N} \mathrm{Al}$ and $7 \mathrm{~N}$ $\mathrm{Ga}$ ) were generated by using the standard ABN $80 \mathrm{DF}$ (Double Filament) effusion cells. The cell shutters are made of pyrolytic boron nitride (PBN) which is relatively highly transparent for IR radiation. The shutters are intended to minimize the overheating of the effusion cells, and thus to minimize the so-called "burst" effect of the generated molecular flux once the cells are opened. Even then, however, the "burst" effect is not completely eliminated. The beam of $\mathrm{As}_{4}$ molecules was produced by the valved-cracker As effusion cell. The calibration of needed molecular fluxes was performed by the use of BayardAlpert gauge.

The growth processes were realized automatically, by the computer procedures. The temperature profiles for effusion cells and for substrate were controlled by a set of thermocouples and Eurotherm controllers.

The set of UHV pumps, consisting of the ion pump, titanium sublimation pumps, as well as cryogenic pump, has been used for pumping of the reactor volume. During the epitaxial runs the sublimation pump has been routinely turned off. The other pumps were operated continuously. The purity of the reaction zone was controlled continuously by a quadrupole mass spectrometer, and additionally by a Bayard-Alpert gauge.

A very important for the technology development was the ability to predict theoretically the properties of the structures with varied design as well as the possibility to evaluate the influence of a particular design feature on the lasing ability of the devices. The above was provided by the theoretical model of the device electronic band structure. The appropriate software package has been developed in Matlab. It allows for calculating relevant properties of the structures depending on their geometry and applied electric field. The possible calculations include: solving Schrödinger equation for energies and wave functions, as well as calculating dipole matrix elements, LO phonon scattering rates, lifetimes and transition rates. The software was tested by comparing results of calculations for structures published in the literature with reported data. The selected results were also compared with the ones obtained by using nextnano ${ }^{3}$ package [6]. The software package was primarily used for calculation of the parameters of the planned laser construction. Next, during the epitaxial technology development it has been intensively used for better understanding of the electrical properties of the achieved QCL structures, and for linking them with the measured constructional parameters, and thus for optimization of the QCL technology.

The high resolution X-ray diffractometry (HRXRD) was used as a method of verifying the constructional assumptions, concerning the thicknesses and compositions of layers. The measurements have been performed 
by X'pert PRO PANalytical diffractometer. The X-ray diffractometry has been used both for in depth study of QCL properties as well as for on-line examination of grown structures and has been found crucial for technology development. The important information have been provided by transmission electron microscopy (TEM) as well as scanning electron microscopy (SEM). They have been used for imaging the cross-sectional profiles of grown heterostructures. Some optical characterization techniques, like photoluminescence (PL) and photoreflectance (PR) have played the role of additional methods for investigation of the superlattice test structures.

The appropriate doping levels of layers constituting the laser structure were confirmed by test based on the Hall effect (including the measurement of concentration and mobility of electrons) made on the specially deposited test structures measured in Van Der Pauw geometry.

\subsection{Technology development}

We have found that the best way to start development of the epitaxial technology of QCL heterostructures is to elaborate at first the most critical element of the device, i.e., the geometry of its active region band structure. As this is governed by the thicknesses and compositions of layers constituting the QCL's AR, the achievement of appropriate precision, spatial uniformity and repeatability of thicknesses/compositions of relevant layers, is the most important issue of the MBE technology, and the device technology at all. Thus the growth of AR layers must definitely be performed in conditions ensuring the maximum control of the above mentioned technological parameters.

The least tolerance of the device for the thickness/composition aspect, points to the growth rates of binaries constituing the layers (i.e., in the case of our elaborated structure the growth rate of GaAs and AlAs), as to the crucial aspect of epitaxial technology. Thus the conditions of growth of AR heterostructure must definitely ensure the maximum precision and stability of every binary growth rate.

To match these requirements in particular the molecular fluxes and incorporation efficiency of impinging atoms into the growing layer have to be appropriately governed. As the flux "burst" effect may disturb the flux control, no growth interruptions between the individual layers within the active region were applied. Hence, only the Al effusion cell was activated and closed during the QCL growth run, according to the need. Thus the "burst" was connected only with the $\mathrm{Al}$ cell, and not with the Ga one. To ensure the ideal stability of the Ga sticking coefficient the relatively low temperature of substrate $\left(T_{\mathrm{S}}\right)$ was applied during the epitaxy of all the heterostructure. The $T_{\mathrm{s}}$ value, controlled on the surface of the growing crystal by a pyrometer, was kept at $580^{\circ} \mathrm{C}$. This was connected with even excessive care, as it is reported that the $\mathrm{Ga}$ sticking coefficient stays nearly unity up to $T_{\mathrm{s}} \approx 630^{\circ} \mathrm{C}$. The value of $\mathrm{V} / \mathrm{III}$ ratio was at least 35 for growth of both GaAs and $\mathrm{Al}_{0.45} \mathrm{Ga}_{0.55} \mathrm{As}$ layers. Thus, in these conditions, the $2 \times 4$ surface reconstruction pattern for GaAs layers was nearly continuously controlled by observation the reflection high-energy electron diffraction (RHEED) image during the growth of structure.

Some errors of the layer thickness resulted from the finite accuracy of the layer growth time, and were related to the time resolution $(0.1 \mathrm{~s})$ realized by software used for controlling the epitaxial runs. As this procedural restriction was unavoidable, the relatively low material growth rate was applied, in order to minimize the errors. The relatively low temperature of $\mathrm{Al}$ effusion cell as needed for low $V_{\mathrm{AlAs}}$ is an additional advantage, because it helps to decrease the effect of cell "burst" on the opening.

As the impinging molecular beams are aligned under the acute angles toward the substrate, the thickness/composition errors may result, because of temporary different flux at different localizations on the surface of growing crystal. To avoid such planar and vertical nonuniformity the substrate was rotated during growth with the speed of $60 \mathrm{rpm}$. This speed was adjusted taking into account the growth rate and the thickness of the thinnest layer of the active region.

The GaAs growth rate $V_{\mathrm{GaAs}}$ was adjusted in situ by RHEED intensity oscillations as equal to $0.5 \mathrm{ML} / \mathrm{s}$. The growth rate $V_{\mathrm{AlAs}}$ of AlAs was adjusted to match the $45 \%$ of this binary in AlGaAs barriers. The appropriate values of $V_{\mathrm{GaAs}}$ and $V_{\mathrm{AlAs}}$ were found by RHEED intensity oscillations just before the every deposition process. The corresponding gallium and aluminum fluxes were applied during the growth of the whole QCL heterostructure. The stability of $V_{\mathrm{GaAs}}$ and $V_{\mathrm{AlAs}}$ was subsequently checked by the RHEED oscillations method just after the epitaxy process. The correctness of $V_{\mathrm{GaAs}}$ and $V_{\mathrm{AlAs}}$ callibrations was also confirmed ex situ by HRXRD on test superlattices.

The on-line HRXRD measurements have been found to be really crucial for QCL technology development. To obtain information about the thickness and composition of component layers of both test structures and complete QCL structures, a theoretical analysis of double-crystal rocking curves was carried out. The dynamical diffraction theory has been used for simulating the symmetric (004) reflections, leading to the extraction of structural parameters of periodic structures. Detailed analysis of measured HRXRD triple-axis $2 \theta / \omega$ scans (see Fig. 3) documents almost perfect agreement between optimized laser structure and simulated one referring to the intended design. This is seen from matching the measured and calculated satellite peak positions, as well as the positions of peaks corresponding with the mean composition of the heterostructure. About 1\% thickness accuracy has been routinely achieved for lasing structures. The barrier layers have been found to contain $(45 \pm 1) \%$, which is, however, not that critical for lasing as the possible thickness inaccuracy. All the above mentioned features of diffraction curves, and particularly the numerous and narrow satellite peaks document strict periodicity of the structures. 

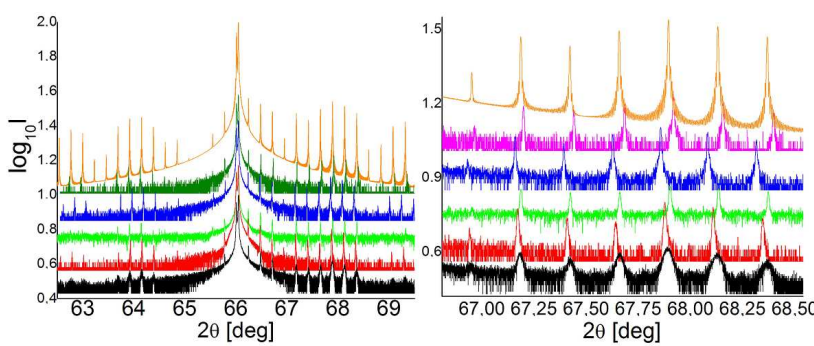

Fig. 3. Collection of HRXRD $2 \theta / \omega$ curves for a series of QCL heterostructures grown in IET; the top curve is a simulation for the planned structure.

Figure 3, presenting the collection of HRXRD profiles recorded for the grown series of QCL structures, documents the needed precision, stability, and run-to-run reproducibility of the used MBE technology.

The perfect planar uniformity of the grown structures was proved by the PL and PR measurements, performed for a set of locations on the SL test structure [7, 8]. The full agreement of the shape of corresponding experimental curves with theoretical predictions is presented in Fig. 4. The periodicity of the AR structure has been additionally confirmed by TEM results (see Fig. 5). However, the precision of thickness determination from TEM was seemingly lower than the one obtained from HRXRD measurements, and hence cannot state the enough precise base for the technological calibrations. It has to be stressed here that failed geometry (a few percent error in module thickness) resulted in the enhanced current leakage, and the lack of lasing [9].

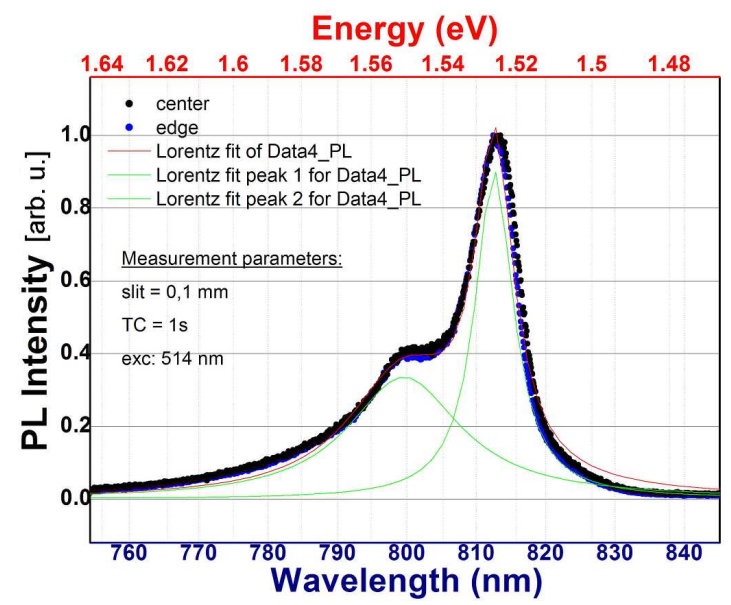

Fig. 4. PL experimental curves obtained for a set of locations on the SL test structure (SL $100 \times$ AlGaAs/GaAs, room temperature (RT) - by the full agreement of the corresponding curves the perfect planar uniformity of the grown structures is proved.

Apart from the X-ray diffraction data, providing directly the constructional parameters of the heterostructures, the final proof of correctness of device geometry

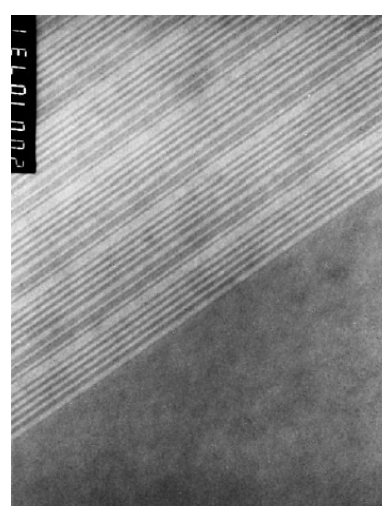

Fig. 5. TEM picture of the few periods of QCL active region. Let us note the periodical construction of the AR [13].

was provided by electrical measurements performed on processed test devices. The correct current-voltage characteristics, i.e., obtained for the heterostructure in which the thicknesses and compositions of AR layers were properly engineered, are characterized by unique features (see Fig. 6). At low bias QCL structures should be highly resistive. When the electric field reaches the value for which the alignment of the pumped laser state and the injector's states occurs, electrons start flowing through the device. In this regime the operating voltage increases linearly with injection current. The saturation of the $I-V$ characteristics, i.e. the high differential resistance of the device, is caused by the onset of misalignment between the upper laser level and the injector ground state. The above given description should match the result of $I-V$ measurement performed at low temperature $(77 \mathrm{~K})$; for higher temperatures the lower values of device differential resistance in all current regimes are observed as a rule.

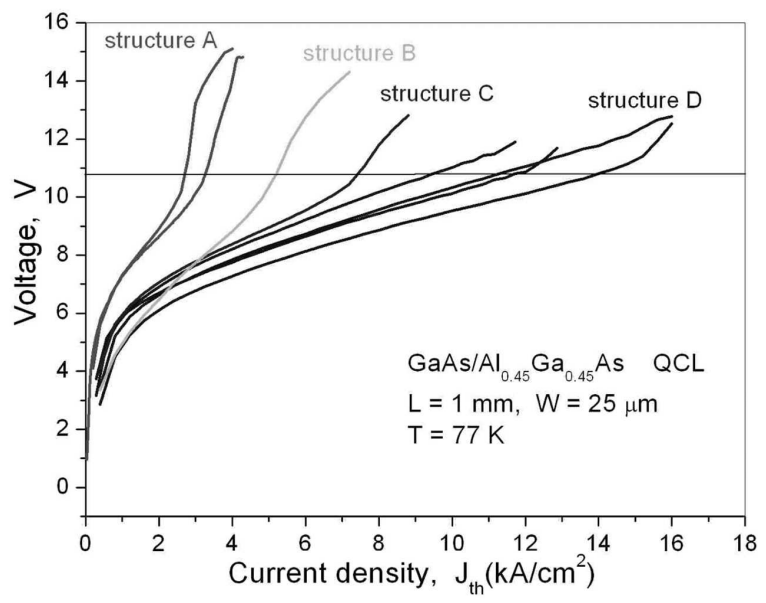

Fig. 6. Voltage-current characteristics for a set of $\mathrm{Al}_{0.45} \mathrm{Ga}_{0.55} \mathrm{As} / \mathrm{GaAs}$ QCLs with different injector doping level $\left(\approx 0.4-\approx 2.2 \times 10^{12} \mathrm{~cm}^{-2}\right.$ per period $)$; the doping concentration increases from structure A to D. 
The effect of saturation, limiting the dynamic range of laser operation $[10,11]$ depends on the injector doping concentration. As the threshold condition may be reached only after the gain exceeds the losses, it is possible that the saturation condition may appear before the losses are exceeded. That is why to achieve lasing the high enough currents must be generated before the saturation, and this requirement, as dependent on injector doping, must be fulfilled experimentally for a given case. The injector doping optimization was just the next step of our QCL technology development, after the proper device geometry was ensured. As the initially applied injector doping level $\left(\approx 0.4 \times 10^{12} \mathrm{~cm}^{-2}\right)$ was found to be too low to achieve lasing, the wide range of injector doping was tested for a fixed QCL geometry. It turned out that much higher currents before the saturation were necessary for lasing, and hence the injector doping had to be increased substantially (at least to $\approx 10^{12} \mathrm{~cm}^{-2}$ ).

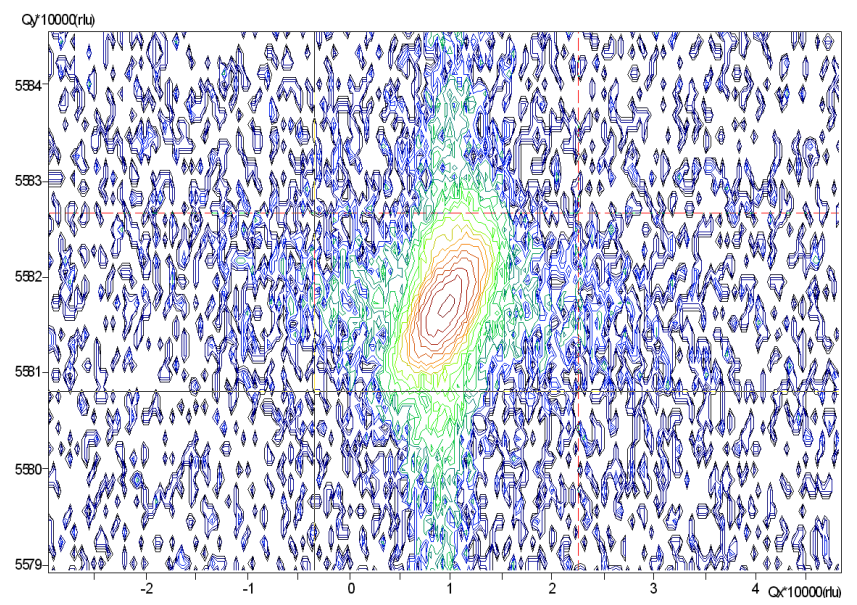

Fig. 7. X-ray reciprocal space map of the selected satellite peak, measured for QCL structure.

Though the lasing effect was achieved as a result of the presented above development of epitaxial technology, it has to be stressed that the resulting devices were not fully optimized. The present state of technology is a starting point for such optimization with respect to the given device parameter. The most immediate way of improvement is the AR interfacial morphology and overall purity of the layers. The growth front of $\mathrm{Al}_{0.45} \mathrm{Ga}_{0.55} \mathrm{As}$ layer may be particularly roughened in comparison with the AlGaAs with lower Al content. The interfacial roughness and foreign atoms may result in enhanced carrier scattering, accompanied by transitions from injector directly to the lower laser level. This also means that it will be necessary to check the increased substrate temperature $T_{\mathrm{s}}$ in the context of increasing the interfacial smoothness and decreasing the concentration of foreign atoms, as well.

The careful adjustment of the $\mathrm{V} / \mathrm{III}$ flux ratio necessary for growth of different regions of the QCL heterostructure may efficiently improve the growth front smoothness, and create the better starting conditions for the growth of device active region. This conclusion is based on the observation that for the proper growth of the waveguide region the lower V/III flux ratio is necessary than the one optimum for the growth of AR. The high amount of adsorbed As atoms is the reason of the decreased mobility of group III elements adatoms. Hence, the resulting excess of As, used for growth of waveguides in our structures, probably increased the roughness of the growth front before the AR epitaxy.

On the other hand, the method of improvement of the material purity by increasing the growth rate $\left(V_{\mathrm{gr}}\right)$ is not useful here. This is because of the detrimental effect of high $V_{\text {gr }}$ on the possibility of meeting the constructional requirements (as discussed above), and on the interfacial smoothness as well.

HRXRD technique gave also some information about other structural properties of the grown crystals, e.g., on the basis of the X-ray reciprocal space map, the absence of any extended defects or strain in the studied QCL heterostructure is believed (Fig. 7).

\section{QCL devices}

\subsection{Device processing and characterization}

The grown QCL heterostructures have been processed into complete devices [12]. In order to limit the lateral current spreading, the double-trench constructions were fabricated. They have been processed by standard processing technology, i.e. photolithography and wet etching $\left(\mathrm{HCl}: \mathrm{H}_{2} \mathrm{O}_{2}: \mathrm{H}_{2} \mathrm{O}=40: 4: 1\right)$. The trenches have been etched only slightly under the AR, i.e. they only shallowly penetrated the lower part of the waveguide's core (Fig. 8). The obtaining of good quality (i.e.

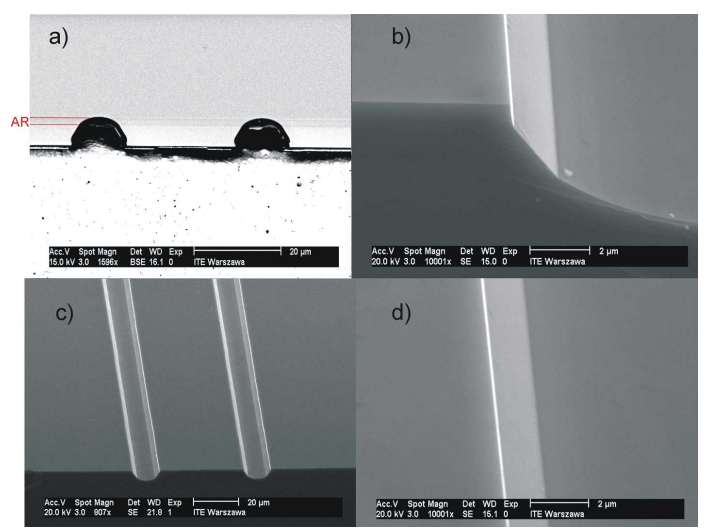

Fig. 8. Scanning electron micrographs of the double trench lasers formed by wet etching; the etching process stops on bottom waveguide level (a); there is no any roughness seen on the edge of waveguide (b)-(d).

smooth) sidewalls, in order to avoid the scattering losses at the waveguide edges, was found to be extremely im-

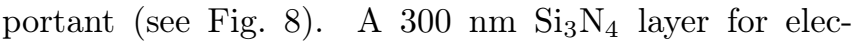
trical insulation was grown by plasma enhanced chem- 

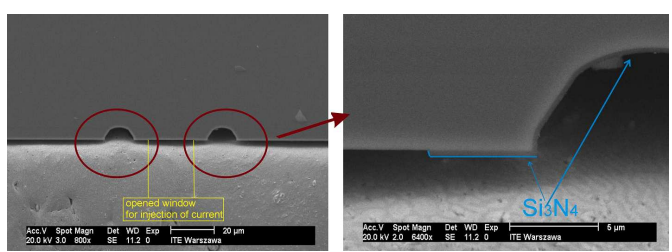

Fig. 9. Scanning electron micrograph of the double trench lasers with dielectric isolation.

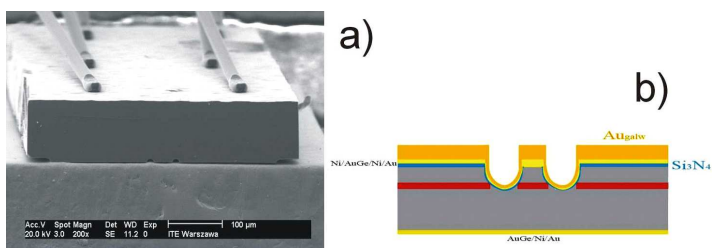

Fig. 10. $\mathrm{Al}_{0.45} \mathrm{Ga}_{0.55} \mathrm{As} / \mathrm{GaAs}$ quantum cascade laser made in IET (a) and cross-section of the device (b).

ical vapor deposition (PECVD). The windows for current injection with widths of 25,35 , and $50 \mu \mathrm{m}$ were opened, with left two $5 \mu \mathrm{m}$ wide stripes on the edges of the ridge (see Fig. 9), for better current injection control. The $5 \mathrm{~nm} \mathrm{Ni} / 100 \mathrm{~nm} \mathrm{AuGe/35} \mathrm{nm} \mathrm{Ni/300} \mathrm{nm} \mathrm{Au}$ $n$-type electric contact metallization structure, alloyed in $430^{\circ} \mathrm{C}$, has been made on top of the heterostructures, with additional Ni layer (when compared with commonly used $\mathrm{AuGe} / \mathrm{Ni} / \mathrm{Au}$ structure) placed directly on GaAs for metal adhesion improvement and for diffusion barrier for $\mathrm{Ge}$ and $\mathrm{Au}$ formation [13]. The average specific contact resistance was $\approx 6 \times 10^{-7} \Omega \mathrm{cm}^{-2}$. An alloyed $\mathrm{AuGe} / \mathrm{Ni} / \mathrm{Au}$ contact was deposited on the wafer's backside, after it was thinned to $\approx 100 \mu \mathrm{m}$. The wafers have been cleaved into bars of 1 and $2 \mathrm{~mm}$ length and the resultant individual devices have been soldered epitaxial side down directly on diamond heatspreaders covered by $\mathrm{Au} / \mathrm{Sn}$ eutectic, and next mounted on copper submounts (Fig. 10).

\subsection{Properties of the lasers}

The emission of lasers was recorded with thermoelectric (TE) cooled $\mathrm{HgCdTe}$ detector (the detector active area was $0.3 \times 0.3 \mathrm{~mm}^{2}$ ). Light from the laser was shined directly on the detector, without collimating lens.

The basic electrical characterization was carried out in the temperature $(T)$ range from $77 \mathrm{~K}$ to $300 \mathrm{~K}$. The lasing has been detected up to the temperature of $\approx 240 \mathrm{~K}$ (for high filling factor of $0.1 \%$, and long pulses of $2 \mu \mathrm{s}$ ), above which it have declined (Fig. 11). When supplied with the 200 ns current pulses the lasing record temperature achieved was $262 \mathrm{~K}\left(-11^{\circ} \mathrm{C}\right)$. For the $1 \mathrm{~mm} \times 25 \mu \mathrm{m}$ device operateing in pulse mode, the optical power in the range of $230-240 \mathrm{~K}$ was between 40 and $10 \mathrm{~mW}$. This fact should be highlighted as it means the possibility of work on the TE cooler. The elimination of the necessity of cryogenic cooling makes the device practical for field applications. For the best lasers the lasing power dropped as a function of temperature with the parameter $T_{0}=$ $310 \mathrm{~K}$ (for $T<140 \mathrm{~K}$ ) and $T_{0}=284 \mathrm{~K}$ (for $T>140 \mathrm{~K}$ ). The related issue is the decrease of emitted optical power for increasing filling factor as seen in Fig. 12.

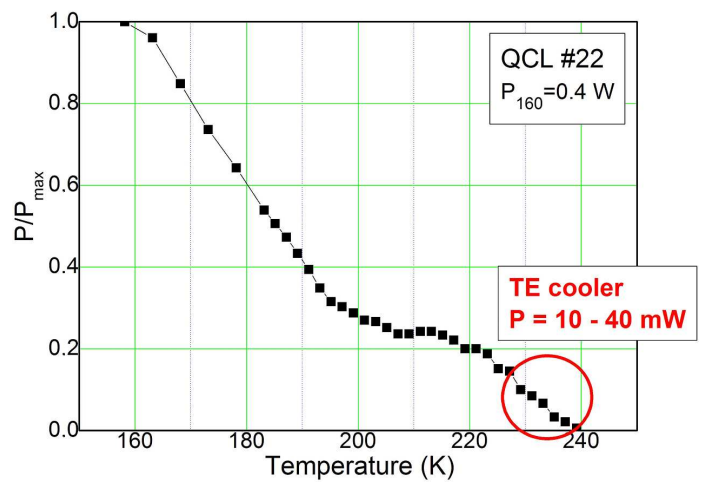

Fig. 11. The dependence of device's optical power drop on temperature; the range of possible application of TE cooler is marked.

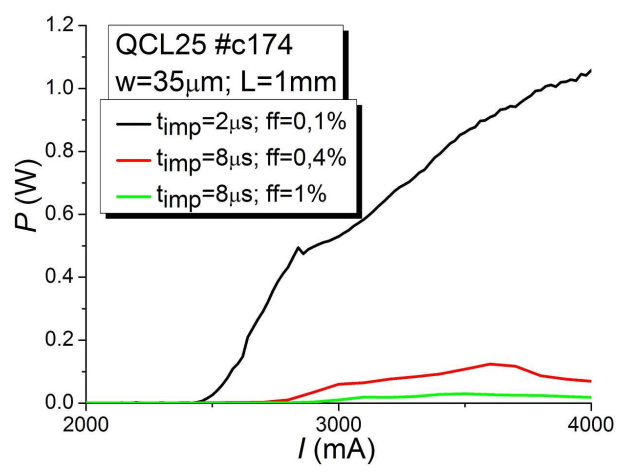

Fig. 12. The comparison of QCL's $P-I$ characteristics for different filling factors.

The record pulse optical power measured at $77 \mathrm{~K}$, for laser without mirror coatings was $1.14 \mathrm{~W}$. The corresponding optical slope efficiency was $0.57 \mathrm{~W} / \mathrm{A}$, and the threshold current density was $7 \mathrm{kA} / \mathrm{cm}^{-3}$. The emission wavelength measured at $77 \mathrm{~K}$ was $9.4 \mu \mathrm{m}$. In Fig. 13 a pair of corresponding light-current $(P-I)$ and currentvoltage $(I-V)$ characteristics is presented.

As it was mentioned above, the three different regions are distinguishable in the current-voltage characteristic, i.e. the region for low polarization before alignment of bottom injector state with the upper laser state, for the properly aligned states, and for the subsequent misalignment at excessive polarization. They are the most clearly visible for the lowest temperature of measurement $(77 \mathrm{~K})$ (Fig. 14). The resistance for the low polarization region significantly decreases with increasing temperature (see Fig. 14). It is clear that with temperature increase spreading of electrons over the available energy states increases, and the leakage of carriers through the quasi- 


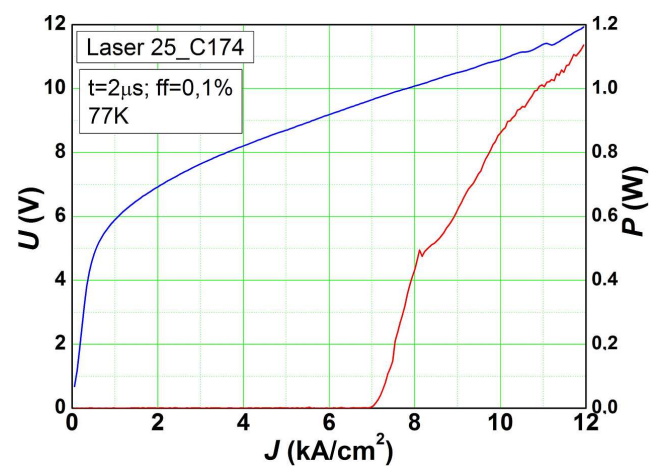

Fig. 13. Light output and current-voltage characteristics; the devices operated at $77 \mathrm{~K}$ in pulsed mode with powers up to $1 \mathrm{~W}$ per uncoated facet; the GaAs based QCLs emitting at $9.4 \mu \mathrm{m}$, made in IET.

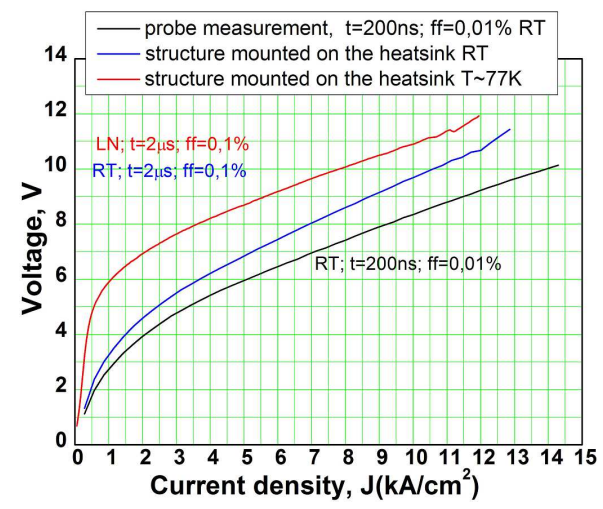

Fig. 14. The comparison of QCL's $I-V$ characteristics for $77 \mathrm{~K}$ and RT.

continuum contributes to the total device current. The increased doping of the injector regions elongates the dynamic region, which may be highly exploited in lasers technology. Also the dynamic resistance of the saturation region significantly decreases with increasing doping level (see Fig. 6). Statistically the highest optical powers as well as the slope efficiencies were obtained for the lasers with the highest doping of injectors.

\section{Conclusions}

The development of molecular beam epitaxy technology of high quality AlGaAs/GaAs structures has been presented. The influence of technological conditions on the structure properties was presented and discussed in depth. The fabrication of AlGaAs/GaAs based QCLs emitting at $9 \mu \mathrm{m}$, being the ultimate test of technology, is reported. The devices operated in pulsed mode at up to $260 \mathrm{~K}$. The peak powers recorded in $77 \mathrm{~K}$ were over $1 \mathrm{~W}$, and the slope efficiency $\eta \approx 0.5-0.6 \mathrm{~W} / \mathrm{A}$ per uncoated facet. These results are fully comparable with the state of the art devices of similar design produced in other laboratories.

\section{Acknowledgments}

The authors would like to acknowledge L. Ornoch, for help in the electrical measurements, P. Romanowski and I. Sankowska for XRD measurements, A. Wójcik-Jedlińska and K. Pierściński for optical measurements, and Adam Łaszcz for TEM imaging. The work was financially supported by grant PBZ-MIN-02/I/2007.

\section{References}

[1] C. Sirtori, GaAs Quantum Cascade Lasers: Fundamentals and Performance, EDP Sciences, 2002.

[2] C. Gmachl, F. Capasso, D.L. Sivco, A.Y. Cho, Rep. Prog. Phys. 64, 1533 (2001).

[3] H. Page, C. Becker, A. Robertson, G. Glastre, V. Ortiz, C. Sirtori, Appl. Phys. Lett. 78, 3529 (2001).

[4] C. Sirtori, P. Kruck, S. Barbieri, P. Collot, J. Nagle, M. Beck, J. Faist, U. Oesterle, Appl. Phys. Lett. 73, 3486 (1998).

[5] P. Harrison, Quantum Wells, Wires and Dots: Theoretical and Computational Physics, 2nd ed., Wiley, Chichester, U.K. 2005.

[6] http://www.nextnano.de .

[7] A. Wójcik-Jedlińska, M. Wasiak, K. Kosiel, M. Bugajski, Opt. Appl., in press.

[8] M. Motyka, G. Sęk, F. Janiak, K. Ryczko, J. Misiewicz, K. Kosiel, M. Bugajski, Opt. Appl., in press.

[9] K. Kosiel, J. Kubacka-Traczyk, P. Karbownik, A. Szerling, J. Muszalski, M. Bugajski, P. Romanowski, J. Gaca, M. Wójcik, Microelectron. J. 40, 565 (2009).

[10] S. Hofling, V.D. Jovanovic, D. Indjin, J.P. Reithmaier, A. Forchel, Z. Ikonic, N. Vukmirovic, P. Harrison, Appl. Phys. Lett. 88, 251109 (2006).

[11] Ch. Mann, Q. Yang, F. Fuchs, W. Bronner, K. Kohler, J. Wagner, IEEE J. Quantum Electron. 42, 994 (2006).

[12] K. Kosiel, M. Bugajski, A. Szerling, J. Kubacka-Traczyk, P. Karbownik, E. Pruszyńska-Karbownik, J. Muszalski, A. Łaszcz, P. Romanowski, M. Wasiak, W. Nakwaski, I. Makarowa, P. Perlin, Photon. Lett. Poland 1, 16 (2009).

[13] P. Karbownik, A. Barańska, A. Szerling, W. Macherzyński, E. Papis, K. Kosiel, M. Bugajski, M. Tłaczała, R. Jakieła, Opt. Appl., in press. 